\title{
From Mesoscopic to Nanoscopic Surface Structures: \\ Lithography with Colloid Monolayers
}

\section{By Frank Burmeister,* Claudia Schäfle, Bettina Keilhofer, Clemens Bechinger, Johannes Boneberg, and Paul Leiderer*}

\section{Infroduction}

A current trend in research is the fabrication and charac. terization of smaller and smaller surface structures approaching dimensions of a few nanometers. They are expected to exhibit a number of new physical and chemical properties when compared to micrometer-sized structures of the same material. These can include, for example, magnetic, optical, or catalytic properties.

As conventional lithographic techniques are limited either by the wavelength of light or because they are serial in nature and costly (e.g., e-beam lithography), a series of new and unconventional approaches to nanofabrication have been put forward. Among these, a technique in which submicroscapic colloidal particles are used as a mask for, for example, elching or vacuum deposition, has proved more and more successful, especially in applications where a periodic arrangement of the structures is required. This technique, which has already been dubbed "natural lithography" "[1] or "nanosphere lithography", [2] works in principle as follows: by some means colloidal particles of equal size and shape (normally spherical) are brought onto the surface to be structured. The parlicles usually arrange themselves randomly. However, under favorable conditions, they form hexagonally arranged, close-packed arrays in a self-assembly process. The surface is subsequently exposed, for example, to an ion beam or light. Vacuum deposition is also possible (see Fig. 1). Afterwards, the particles are removed by a lift-off process. Different kinds of structures can then be observed: ion beam etching usually leaves isolated posts of the substrate material, whereas vacuum deposition leads to holey thin films (for random arrangement) or, in the case of a regular arrangement, to the formation of triangular-shaped, elevaled structures arranged in a hon-

[*] F. Burmeister, Prof. P. Leiderer, C Schătle B. Keilhofer Dr. C. Bechinger, Dr. J. Boneberg Department of Physics, University of Konstanz Posifach 5560 M676, D-78434 Konstanz (Germany) eycomb pattern. The formation of ring-like patterns has also been reported recently. ${ }^{[3.4]}$

As suspensions of colloidal particles are commercially available with particle sizes covering three orders of magnitude (10 nm to $10 \mu \mathrm{m}$ ), surface structures of almost any desired size and periodicity can, in principle, be fabricated. Besides the aforementioned possibilities of tundamental research on na-

a)

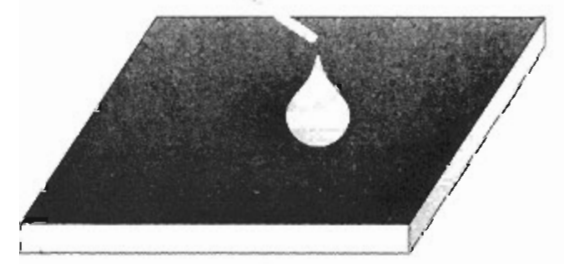

b)

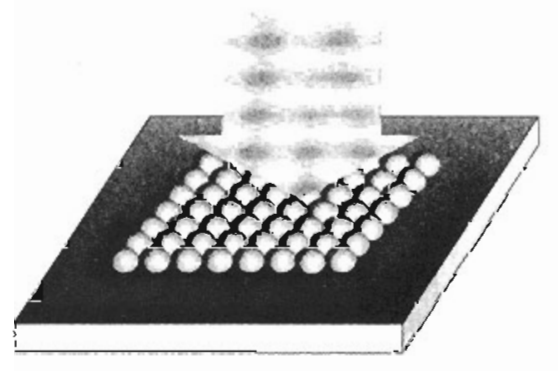

c)

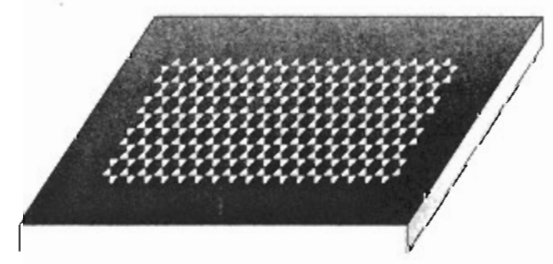

Fig. 1. Principle of colloid monolayer lithography: a) application of suspension, b) deposition of metal onto ordered monolayer, c) reqularly arranged metal suructures on the surface after removal of the particles. 
nostructures, surfaces with defined roughness and periodicity may also find several technological applications, for example, as diffraction gratings, selective solar adsorben, or antireflection coatings ${ }^{[5]}$ In the following, a very brief overview is given of the research activities in this Geld with the main emphasis on new developments and current trends.

\section{Pioneering Work}

Probably the first report in which ordered monolayers of colloidal particles were systematically utilized for the fabrication of submicroscopic surface structures was published in 1981 by Fischer and Zingsheim. ${ }^{[6]}$ They simply applied a suspension of colloidal particles with a diameter of $312 \mathrm{~nm}$ onto a glass plate and allowed it to evaporate. Afterwards, small portions of the glass plate were covered with a monolayer of hexagonally arranged spheres. Vacuum deposition of platinum through the voids between neighboring spheres led to arrays of triangular platinum structures on the surface. The size and the periodicity of the platinum pattems were smaller than the wavelength of visible light.

Shortly thereafter, Deckman and Dunsmuir ${ }^{(1)}$ described new methods for the fabrication of both randomly and regularly arranged roonolayers of particles. They made extensive use of reactive ion beam etching to structure surfaces and demonstrated some technological applicalions of colloid monolayer lithography. ${ }^{[7,8\}}$

\section{Theory of 2D Self-Assembly}

The experiments carried out by Nagayama and cowork$e^{e r s}{ }^{[9]}$ revealed for the first time the mechanism of the selfassembly process that eventually leads to hexagonally close packed amays of colloidal particles. Following their argumentation, array formation proceeds in two steps: First, a nucleus is formed when the thickness of the solvent layer (generally water) approacbes the diameter of the particles. When the tops of the particles protrude from the water surface, it is deformed and, due to surface tension effects, the spheres are pulled together. Theoretical calculations have shown that these lateral capillary forces can exceed the thermal energy $k_{\mathrm{B}} T$ of the particles by many orders of magnitude. ${ }^{[0]}$ In the second step, evaporation of the solvent from within the micromedisci between the spberes causes water influx from outside, which is related to convective particle transport towards the nucleus.

\section{Fabrication Methods}

Numerous methods for the production of colloid monolayers have been proposed in the literature, so only the most promising ones will be discussed. Hulteen and Van Duyne ${ }^{[2]}$ used a spin-coating procedure and, by varying the particle concentration and spin speed, could reproducibly fabricate single and double layers of polystyrene spheres (deposition through a double layer leads to a real hexagonal pattern on the surface witb balf the usual number of structures). Another, very simple method, introduced by Micheletto et al. ${ }^{[11]}$ works as follows; a droplet of the colloid is applied to a carefully cleaned glass plate and the whole is enclosed in an airtight box. The evaporation rate is determined by a control circuit for temperature and ambient humidity. A nucleus is defined just by tilting the glass plate such that array formation starts at the upper edge and proceeds downwards. Monolayers consisting of particles down to $50 \mathrm{~nm}$ in size were successfully fabricated. However, the approach most suitable for industrial applications was suggested by Dimitrov and Nagayama: ${ }^{[.5]}$ a glass plate is vertically dipped into a colloidal suspension and afterwards very slowly withdrawn. Again, temperature and ambient humidity are controlled. The thickness of the drying film, which is formed in a similar manner to one in a sol-gel process, could be adjusted to single, double, or triple layers, etc., just by measuring the optical transmission of the film and adapting the withdrawal speed accordingly. In this way, square-centimeter-sized arrays were reproducibly obtained.

However, there is at least one major drawback 10 all these methods: either the randomness of the crystallization process and/or the need for very smooth and hydrophilic substrates. We recently circumvented these drawbacks: ${ }^{[12]}$ First, we fabricated a colloid monolayer on a glass slide by the above mentioned method of Micheletto et al. ${ }^{[1]}$ It was then stabilized by vacuum deposition of a metal or by thermal annealing. The stabilization caused shrinking of the openings between the spheres without completely blocking them. Afterwards the slide was slowly dipped into a watercontaining vessel and the monolayer gradually floated off as a whole onto the water surface, similar to ultrathin polymer films. The floating particle array was then transferred onto the desired surface. This technique could be further improved when pick-up was performed with a transmission electron microscope (TEM) grid. In this way, a free-standing, transportable lithographic mask was produced. It is depicted in Figure 2, together with the result after deposition of a $30 \mathrm{~nm}$ thick layer of gold. A consequence of this achievement is that the structuriag of alcoost any surface on any desired location is now possible, with feature sizes below $100 \mathrm{~nm}$. The necessary equipment and the very low production costs are in principle affordable to any laboratory or research organization.

\section{Future Trends}

Besides reliability and positioning, other requirements for possible technological applications or even for some optical diffraction experiments are long-range order, i.e., very large crystallites with a given orientation. It might also be interesting to induce crystal symmetries other than simply 
a)

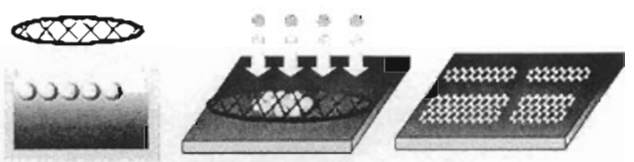

b)

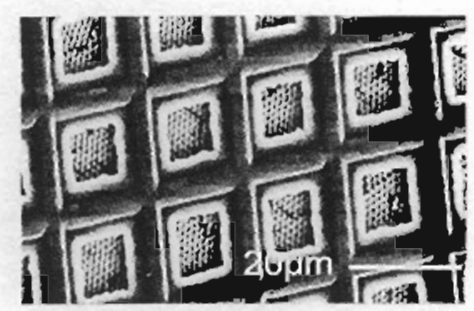

c)

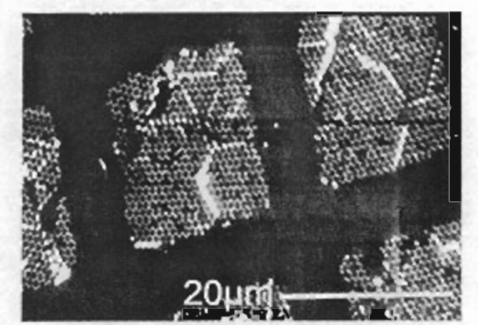

Fig. 2. Application of transportable mask: a) principle of the process (from left to right): TEM grid is used to pick up colloid monolayer, metal is deposited onto desired substrate using the colloid monolayer/TEM grid as a lithographic masik, the mask is remowed to leave regularly arranged metal structures on the surface of the substrate: D) TEM grid covered with a colloid monolayer; $c$ ) deposited gold dots on a highly oriented pyrolytic graphite (HOPG) surface after removal of the mask.

hexagonal. In order to achieve this, one usually employs a prestructured substrate. This was done by Deckman et al. ${ }^{[8]}$ (grapho-epitaxial effect) and, in a certain sense, by Kim et al. ${ }^{[13]}$ (micromolding in capillaries) and by Velev et al., ${ }^{[14]}$ where ail droplets were used as templates for crystallization. We utilized silicon grids as substrates, which wiere fabricated by means of optical lithography. When the period of the grid was commensurate with the hexagonal lattice of the particles, we observed perfect ordering and orientation of the particles (Fig. 3a). However, when the grid period was adjusted to coincide with quadratic packing of the particles, the arrangement was not as perfect as in the hexagonal case (Fig. 3b). One would probably need a substrate prestructured in two dimensions, comparable to the experiments of van Blaaderen et al. ${ }^{[15]}$ (colloidal epitaxy).

Even though the ordering of the particles was not always perfect, we could observe their alignment along the whole length of the grid $(1 \mathrm{~cm})$ quite frequently. Therefore we believe 1 hat, perhaps in combination with other fabrication methods, the production of square-centimeter-sized crystallites of colloid monolayers with predefined orientation should be possible. They will still contain some voids and dislocations, which are mainly due to the inherent polydispersity of the particles, but these minor imperfections are meaningless for both fundamental research on single nanostructures and also for most of the above-mentioned technological applications.

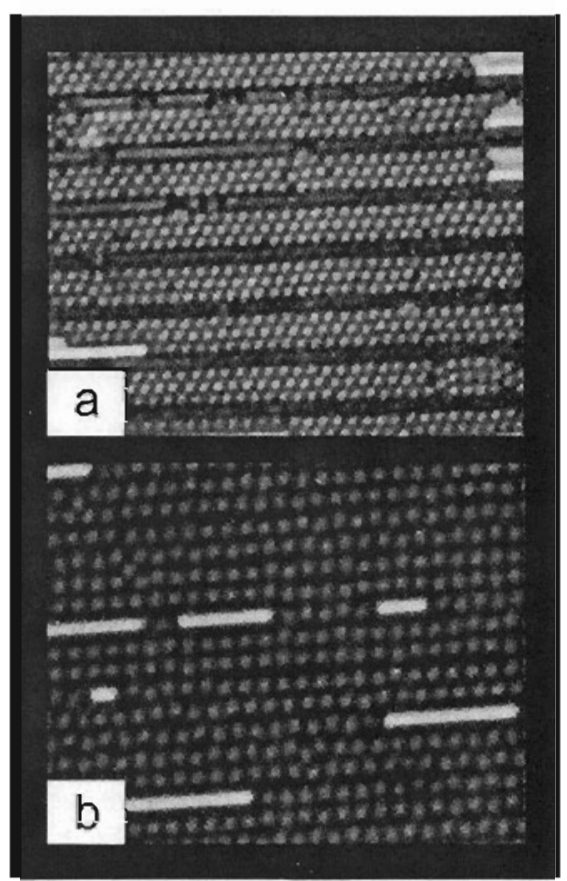

Fig. 3. a) Silicon grid covered with $840 \mathrm{~nm}$ sized spheres: period $3 \mu \mathrm{m}$, depth of urenches $100 \mathrm{~nm}$. b) Same spheres as in (a) but with a grid period of $850 \mathrm{~nm}$ and a trench depth of $110 \mathrm{~nm}$.

\section{Conclusions}

To summarize, we believe that lithography with colloid monolayers has developed into a powerful and alternative technique for the nanostructuring of surfaces. Production of colloid monolayers with $50 \mathrm{~nm}$ sized spheres has already been demonstrated as well as square-centimeter-sized arrays. Numerous applications, together with a vast variety of materials combinations, have been introduced. Major challenges for the future will be the routine utilization of very small paricles and the precise positioning and orientation of particle arrays on a given substrate surface.

11] H. W. Deckman, J. H. Dunsmuir, Appl. Phys Lefr. 1982, 41,377

[2] J. C. Hulteen, R. P. Van Duyne. J. Vac. Sci. Technol. 1995, $\lambda J 3,1533$

[3] M. Winzer, M. Kleiber, N. Dix, R. Wiesendanger, Appl. Phys. A 1996. 63,617 .

[4] J. Boneberg. F. Burmeister, C. Schäfle, P. Leiderer, D. Reim, A. Fery, S. Herminghaus, Langmuir, in press.

[5] A. S. Dimitrov, K. Nagayama, Langmuir 1996, 12, 1303

[6] U. C. Fischer, H. P. Zingsheim, J. Vac. Sci. Technol. 1981, 19.881.

[7] H. W. Deckman, J. H. Dunsmuir, European Patenl EP-0-083510 BI. 1987, Europeain Patent EP-0-270212 B1. 1990.

[8] H. W. Deckman, J. H. Dunsmuir. S. Garoff. J. A. McHenry, D. G Pfeiffer, J. Vac. Sci. Technol. 1988, B6, 333.

[9] N. D. Denkov, O. D. Velev, P. A. Kralchevsky, I. B. Ivanov, H. Yoshiimura, K. Nagayana, Langmuir 1992, 8. 3183.

[10] P. A. Kralchersky, K. Nagayama, Longmuir 1994, 10, 23.

[11] R. Micheletto, H. Fukuda, M. Ohtsu, Langmuir 1995, 11, 3333.

[12] F. Burmeister, C. Schäfle, T. Matthes, M. Böhmisch, J. Boneberg, P. Leiderer, Longmuir 1997, 13, 2983.

[13] E. Kim, Y. Xia, C. M. Whitcsides, Adv Mater. 1996, 8. 245.

[14] O. D. Velev, K. Furusawa, K. Nagayama, Langmuir 1996, 12, 2374.

[15] A. van Blaaderen, R. Ruel, P. Wiltzius Noture 1997, 385, 321. 